\title{
An Overview of Evolutionary Computing for Interpretation in the Oil and Gas Industry
}

\author{
Rui L. Lopes ${ }^{1}$ \\ rui.l.lopes@inesctec.pt \\ Hamed Nikhalat-Jahromi ${ }^{1}$ \\ hnj@inesctec.pt \\ Alípio M. Jorge ${ }^{1,2}$ \\ ${ }^{1}$ Institute for Systems and Computer Engineering, Technology and Science, Porto, Portugal \\ ${ }^{2}$ DCC - FCUP, Universidade do Porto, Portugal
}

\begin{abstract}
The Oil and Gas Exploration \& Production (E\&P) field deals with high-dimensional heterogeneous data, collected at different stages of the E\&P activities from various sources. Over the years different soft-computing algorithms have been proposed for data-driven oil and gas applications. The most popular by far are Artificial Neural Networks, but there are applications of Fuzzy Logic systems, Support Vector Machines, and Evolutionary Algorithms (EAs) as well.

This article provides an overview of the applications of EAs in the oil and gas E\&P industry. The relevant literature is reviewed and categorised, showing an increasing interest amongst the geoscience community.
\end{abstract}

\section{CCS Concepts}

-Applied computing $\rightarrow$ Earth and atmospheric sciences; •Information systems $\rightarrow$ Data analytics; Data mining;

\section{Keywords}

Evolutionary Algorithm, Soft-Computing, Data-Mining, Geoscience, Exploration and Production

\section{INTRODUCTION}

We have entered the era of Big Data (high-dimensional heterogeneous, and often unstructured, data), the Oil and Gas E\&P industry is finally embracing new data-driven methodologies, and "in this case, [data] really is the new oil"[23]. The technological breakthroughs in data transmission, storage, and analysis have enabled a move towards the "digital oilfield" or "data-driven oilfield", in an attempt to reduce the costs and risks of the industry's major expense - drilling for oil. This data-driven approach poses many technological challenges, particularly concerning data management and knowledge extraction. This implies the development of specialised hardware and software, requiring oil

Permission to make digital or hard copies of all or part of this work for personal or classroom use is granted without fee provided that copies are not made or distributed for profit or commercial advantage and that copies bear this notice and the full citafor profit or commercial advantage and that copies bear this notice and the full cita-
tion on the first page. Copyrights for components of this work owned by others than ACM must be honored. Abstracting with credit is permitted. To copy otherwise, or republish, to post on servers or to redistribute to lists, requires prior specific permission and/or a fee. Request permissions from permissions@acm.org.

C3S2E '16, July 20-22, 2016, Porto, Portugal

(c) 2016 ACM. ISBN 978-1-4503-4075-5/16/07 . \$ $\$ 15.00$

DOI: http://dx.doi.org/10.1145/2948992.2949006 companies to either invest in in-house data facilities integrated through all business activities, or to out-source these to data-centred companies[6, 17].

The big players in the E\&P industry are slowly but steadily embracing this change[18]. The data-driven oilfield has stimulated the literature on soft-computing algorithms[36] for oil and gas E\&P, particularly with biologically inspired ones. The most popular algorithms are Artificial Neural Networks (ANN), followed by Fuzzy Logic (FL), and Support Vector Machines (SVM)[21]. Other approaches include Swarm Intelligence, such as Particle Swarm Optimisation and Ant Colony Optimisation, as well as different Evolutionary Algorithms (EAs).

The first EAs were developed in the 1960's[13]. Fogel proposed Evolutionary Programming, evolving populations of computer programs represented by finite state machines, and Rechenberg and Schwefel proposed Evolution Strategies (ES) for the evolution of arrays of real values. Following these Holland developed Genetic Algorithms (GA), using a representation similar to ES, but introducing the Building Block Hypothesis and hence the recombination operators into the algorithm. In the early 1990's Koza proposed Genetic Programming (GP) as a means for the automatic programming of computers. Similarly to EP, GP allows the evolution of programs represented by trees, subjecting the individuals to variation operators.

The different algorithms using Darwinian principles (EP, ES, GA, and GP) were later unified under the umbrella of Evolutionary Computing, and started gaining traction amongst researchers. In particular, new representations and operators have been proposed for GP, of which the most adopted have been Grammatical Evolution (GE), and Cartesian GP (CGP), but there other well-known proposals such as Linear GP (LGP), and Neuro-Evolution of Augmented Topologies (NEAT) [8].

In the remainder of this article the applications of EAs in the Oil and Gas industry will be assessed, in particular those related to the E\&P of these natural resources. In Section 2 the different types of problems are presented, along with the approaches proposed to address them. Finally, Section 3 presents some remarks and discussion on this body of research.

\section{EA'S FOR OIL AND GAS E\&P}

Soft-computing algorithms have been increasingly adopted by the Oil and Gas E\&P practitioners, to assist both the data processing and the interpretation activities. These algorithms have been gradually integrated into 
the modelling/interpretation work-flow, with different applications offering several ready-to-use modules for the field scientists.

Important challenges in this industry[5, 17] include i) the accurate positioning of structures (salt/overthrust), ii) the characterization of laminated sands/shales, iii) accurate depth imaging, iv) distinguishing commercial/noncommercial gas, v) fault detection and classification, vi) environmental issues, vii) exploration in difficult areas, viii) fluid/permeability prediction, and uncertainty quantification, ix) identification and classification of fractures, and $\mathrm{x})$ prediction and evaluation of pressure in reservoirs.

The related articles in scientific journals ${ }^{1}$ indicate the popularity of the algorithms. It is clear from the surveying of this landscape that ANNs, FL, and SVMs are the most popular soft-computing techniques. It is also understood that the most commonly addressed problems are related to reservoir modelling and characterisation, as well as history matching. As an example, Elsevier's Journal of Petroleum Science and Engineering dedicated a whole issue to the forefront research of ANNs applied to reservoirs[2].

Different sources of data are used throughout the literature for the quantitative interpretation challenges mentioned earlier. These can be fit into one of two classes: geophysical and petrophysical data (see Figure 1). In the former the most common are seismic and electro-magnetic surveys, while in the latter is most commonly used well logging and core analysis. The interpreter must analyse both types, though constrained by its availability (for instance, many times the field is not entirely surveyed, and well logging and core analysis data is only available after drilling has commenced/completed). The approaches surveyed in this article are approximately even split in what concerns the data type used, with $60 \%$ of the articles using petrophysical logs and $40 \%$ using geophysical surveys.

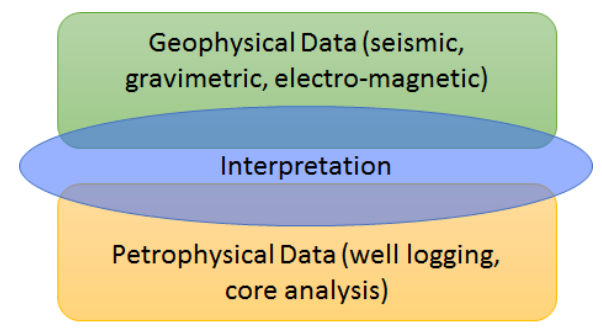

Figure 1: Data types and sources in the data-driven quantitative interpretation process.

Shaheen et al.[31] surveyed data mining applications in hydrocarbon exploration, covering ANNs, FL, and SVMs, addressing as well remote sensing image analysis. The breakdown of the surveyed articles by problem type shows that feature selection is the biggest concern among researchers, with approximately half of the articles dedicated to "influencing factor analysis". The authors also highlighted

${ }^{1}$ Examples of these publications are the Journal of Petroleum Science and Engineering, and the Journal of Natural Gas Science and Engineering (Elsevier), Geophysics (Society of Exploration Geophysicists - SEP), Interpretation (SEP), Energy \& Fuels (American Chemical Society) Artificial Intelligence Review (Springer), IEEE Proceedings, and Society of Petroleum Engineers. reservoir characterisation and well log analysis as commonly addressed problems.

Focusing on real world classification problems from Chinese explorations, Li et al.[24] present a comparative study of five classification algorithms: decision trees, ANNs, SVMs, Bayesian networks, and ensemble learning. Different algorithms for feature extraction were also compared, namely Relief-F and SVM Recursive Feature Elimination (SVM-RFE) for feature ranking, and Correlation-based Feature Selection (CFS) and Las Vegas Filter (LVF), for filter subset selection. Contrasting with the remarks from Shaheen et al.[31], the authors argue that the choice of the classification method should be more important than that of the feature selection algorithms.

Zhao et al.[37] compared supervised and unsupervised algorithms in seismic facies ${ }^{2}$ recognition. They considered cross-plotting, k-means clustering, and different projection techniques - Principal Component Analysis (PCA), Selforganising Maps (SOM), and Generative Topographic Mapping (GTM). Other unsupervised methods were not compared because of the lack of readily available software. Relatively to the supervised algorithms only ANNs and SVMs were addressed. The reported experiments and results show that there is not a single algorithm that is better than the others, but revealed the importance of choosing the attributes appropriately, of using as few attributes as possible, and preferably independent from each other (in line with the remarks from Shaheen et al.[31]).

As mentioned previously, one of the most frequent domains of application of soft-computing techniques is reservoir simulation and modelling. This application was reviewed by Mohaghegh[28], focusing on FL. The author categorised AI-based reservoir models in three categories: Surrogate Reservoir Models (SRM), Top-Down Models (TDM), and Hybrid models (a combination of SRM and TDM). The main advantages of AI-based models reported include the short development time, and minimal computational overhead (which allow for fast track analysis and decision making). The most prominent issues are the databases, which are assembled from different sources and hence are prone to inconsistency, as well as a shortage of trained individuals that can successfully build and analyse these models.

In the early 2000s, Aminzadeh [5] addressed the applications of $\mathrm{AI}$ and soft computing in the oil industry, describing the most promising technologies. Amongst these the authors included Evolutionary Computing (EC), suggesting it could be used to categorise rock formations, to recognise seismic patterns, or to describe the sedimentation process. A decade later EAs still have not been widely adopted. Moreover, its application has revolved around hybrid algorithms where population methods optimise parameters of other soft-computing algorithms. Nevertheless, the application of EC follows the global trend of soft-computing usage in E\&P, with the number of published articles growing in recent years (see Figure 2 for the reference count in this article).

In the remainder of this section the evolutionary approaches found in the literature of oil and gas E\&P, however briefly, are discussed. The methods were divided into

\footnotetext{
${ }^{2}$ Seismic facies are a visual representation of seismic reflection parameters, such as configuration, continuity, amplitude, and frequency, within the stratigraphic framework (rock layers) of a depositional sequence.
} 


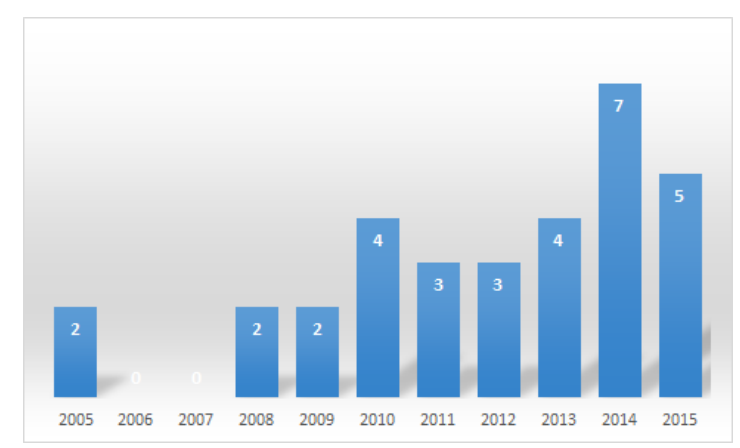

Figure 2: Number of references on Evolutionary Computing in the Oil and Gas E\&P industry cited in this article.

two major categories defined by the nature of the evolved solutions: GAs, and GP algorithms.

\subsection{Genetic Algorithm Approaches}

For most, if not all, of the soft-computing algorithms, there is a set of problem-dependent parameters that affects its performance and is dependent on the experimentation by the practitioners in order to setup (sub)optimal parameters[7]. This is the niche that has been addressed with GAs in the literature, as will be demonstrated in the next paragraphs.

Before an exploratory drill is started, interpreters must rely on their experience and different sources of noisy and imprecise data in order to identify the reservoirs and estimate the ideal location for a well. One of the tools at their disposal is the modelling and simulation of the reservoirs, that involves the characterisation of the reservoir and the construction of a computational model, to improve the estimation of the reserves and the decisions regarding the development of the field.

One of the main tasks found in the literature is to predict the permeability and porosity of rocks. This has been done using different techniques, with GAs being used to optimise the parameters of other predictors such as the weights in ANNs [19], or the coefficients in FL and SVMs [1, 25]. A slightly different application example is to use the GA as an optimiser to find the best logs for prediction of permeability [16], that is, to select the data that will render the best estimator.

The estimation of the reservoir thickness is also important to support the decision of the well drilling point for a given zone. Du and Cheng[12] developed a GA-BP (backpropagation) fuzzy neural network to extract the seismic reservoir fuzzy rules, that is, a system that can convert seismic attributes and reservoir thickness data into fuzzy rules with the form of expert knowledge effectively. Another approach is to use well-logging data for the determination of the thickness of the formation layers, through an inversion (estimating models of physical property distributions based on geophysical survey data). Dobróka and Szabó[11] developed an automated inversion procedure for this task, optimised by a GA.

To fine-tune the reservoir models (physics- or data-based) and reduce the uncertainty, these have to be correlated with well data, when available. The most common approach is to use well logging data, collected during the perforation, but there are approaches in the literature that use well test data - flow rate data, pressure data, and other. This correlation technique is typically called history matching because one is comparing the model output with actual production data from the well.

History matching (and optimisation problems in general) often involve several, potentially conflicting, objectives. For instance, one might seek to minimise a function involving differences in reservoir pressure, multiphase production history, and 4D time-lapse seismic data, which do not always change in a similar fashion. It is common to treat these differences as a single objective optimisation problem by aggregating all objectives into a scalar function, possibly rendering an incomplete exploration of the solution space. Two examples of such approach are described by Labani et al.[22], where the authors used a committee machine with intelligent systems (CMIS) combining FL, Neuro-Fuzzy(NF) and ANN algorithms, for overall estimation of the nuclear magnetic resonance (NMR) log parameters from conventional well log data. They used a GA to optimise the weights of the different algorithms (and hence their contribution) in the CMIS. Other approaches include a Hybrid GA with Least Absolute Value (LAV) local search for the analysis of the decline rate of a fractured gas well[35]; a GA with Adaptive Bounds and distributed simulation to improve the models[26]; or a GA to evolve the weights and thresholds of an ANN for the prediction of fracture development of the target reservoir[34].

In order to improve the consistency and accuracy of the reservoir models with both geological and geophysical measurements, instead of using an aggregate fitness function some researchers have turned to Multi-objective optimisation (also known as Pareto optimisation). Park et al.[30] developed a Multi-objective Evolutionary Algorithm (MOEA) based on NSGA-II ${ }^{3}$ and compared the approach with the traditional (aggregated fitness) GA, demonstrating improved results. Another MOEA based in NSGA-II is presented by Niri and Lumley[14], where the authors simultaneously try to match multiple datasets including well logs, geologic information, and various seismic attributes. Similarly, Min et al.[27] used the NSGA-II algorithm and addressed some of its limitations by introducing preferenceordering (through Dynamic Goal Programming) and successive objective (dimension) reduction (through Successive Linear Objective Reduction). The first prunes any unqualified geomodels (enhancing the convergence speed towards the Pareto-optimal front), and the second excludes redundant production data from the fitness evaluation (improving the optimisation efficiency).

It is clear from the above reviewed works, that the dimensionality of the data (both in number of records and of variables) that needs to be processed and analysed in the various stages of the E\&P activities calls for ensembles of algorithms, tailored for the various sub-problems, or as is the case most frequently with GAs, tailored to optimise other data science algorithms.

\subsection{Genetic Programming Approaches}

The uncertainty inherent to the surrogate models and the design possibilities of the different soft-computing techniques that are themselves used to build those models opens another space for further improvement of the tools at the

${ }^{3}$ Non-dominated Sorting Genetic Algorithm-II [10] 
disposal of exploration engineers and scientists. This could be a niche addressed by GP, but to the best of the authors knowledge the application of these algorithms has not gained much traction, perhaps because its implementation is not as straightforward as with the GAs.

Similarly to the work with GAs, Xie et al.[33] developed a hybrid GP Neuro-Fuzzy algorithm that provides permeability estimates for all rock-types or lithologies. The system is composed of three modules: the first module is an interactive classification of lithology types; the second module is based on Genetic Programming, and was designed to predict permeability facies within the lithology types (corresponding to a categorical classification into low, medium or high permeability set associated with each lithology type); finally, the third module of the system is a neuro-fuzzy inference algorithm, in which an actual permeability value is estimated. Kaydani et al.[20] implemented a multi-gene $\mathrm{GP}^{4}$ to evolve an equation for permeability prediction based on well log and core experimental data.

There are a few examples particularly for the natural gas domain. AlQuraishi et al.[4] developed a GP system for estimating the gas viscosity and density; a GP-based model for the dewpoint pressure in gas condensate reservoirs was implemented by Shokir[32]; a new correlation for the z-factor was inferred by Al-Anazi et al.[3]; and Parhizgar et al.[29] modelled the vaporization enthalpies of petroleum fractions and pure hydrocarbons using GP.

Cranganu and Bautu[9] used Gene Expression Programming (GEP) $)^{5}$ to synthesize missing sonic (DT) logs when only common logs (such as natural gamma ray, or deep resistivity) are present. Large real world data sets were used, and the authors argue that GEP does not over-predict mean values as happens with other non-linear regression techniques and therefore it "preserves original data variability".

The contribution of the GP algorithms in the Oil and Gas industry is not very extensive. However, the works reported above suggest that its application for specific tasks, embedded in other soft-computing work-flows, could prove very rewarding. The final section of this article extends the authors remarks on this literature collection and provides guide-lines for future research on these methodologies.

\section{CONCLUSION}

Different intelligent algorithms have been integrated over the years into the processing and interpretation work-flows in the E\&P industry, automating simple tasks and improving the support to the decision makers. The most common approach is to integrate biologically inspired algorithms into the modelling and simulation of reservoirs. In particular ANNs, FL, and SVMs have gained the most traction in this industry. On the other hand, population-based heuristics have been rarely adopted. These require bigger computational effort, which in conjunction with the highdimensionality of the data may discourage their usage. Nevertheless, GAs have found a niche as an optimiser for other soft-computing algorithms. The examples of GP algorithms are very scarce, even though a couple of recent develop-

\footnotetext{
${ }^{4}$ In Multi-gene GP the model output is a weighted sum of the output of various trees, with the coefficients inferred from data using the Least Squares method.

${ }^{5}$ An evolutionary algorithm similar to GP [15] with a linear genotypic representation
}

ments have been integrated by some researchers (for instance, Multi-gene GP, and GEP).

The possibilities for GP applications in this field are immense, and have barely been tapped. For instance, following the logic commonly found in GAs applications, with GP algorithms one could evolve both the structure and the weights of ANNs, the rules and coefficients of fuzzy systems, or perform feature extraction/selection. Moreover, there is a set of recent algorithms that could add value, and that have been used for other problems with high-dimensional data (CGP, GE, NEAT, amongst others).

The Oil and Gas E\&P industry has entered the new datadriven era in a bid to optimise production and reduce costs. The complexity of the problems and the high-dimensionality of the data require powerful algorithms (and hardware), and soft-computing has an appropriate set of tools at the disposal of the practitioners. However, it remains necessary to bring data scientists closer to geophysicists in order to speed-up these developments. For true knowledge integration, data scientists need better understanding of the geophysics, as much as the geophysicists need better understanding of how data science can assist their activities and research.

\section{Acknowledgments}

This work is financed by the ERDF - European Regional Development Fund through the Operational Programme for Competitiveness and Internationalisation - COMPETE 2020 Programme within project POCI-01-0145-FEDER-006961, and by National Funds through the FCT - Fundação para a Ciência e a Tecnologia (Portuguese Foundation for Science and Technology) as part of project UID/EEA/50014/2013.

\section{REFERENCES}

[1] M.-A. Ahmadi, M. R. Ahmadi, S. M. Hosseini, and M. Ebadi. Connectionist model predicts the porosity and permeability of petroleum reservoirs by means of petro-physical logs: Application of artificial intelligence. Journal of Petroleum Science and Engineering, 123:183-200, 2014.

[2] T. Aifa. Neural network applications to reservoirs: Physics-based models and data models. Journal of Petroleum Science and Engineering, 123:1-6, 2014.

[3] B. D. Al-Anazi, A. Al-Quraishi, et al. New correlation for z-factor using genetic programming technique. In SPE Oil and Gas India Conference and Exhibition. Society of Petroleum Engineers, 2010.

[4] A. A. AlQuraishi, M. A. Jumma, et al. Determination of gas viscosity and density using genetic programing. In Offshore Mediterranean Conference and Exhibition. Offshore Mediterranean Conference, 2009.

[5] F. Aminzadeh. Applications of ai and soft computing for challenging problems in the oil industry. Journal of Petroleum Science and Engineering, 47(1):5-14, 2005.

[6] R. Bertocco and V. Padmanabhan. Big data analytics in oil and gas. Bain Brief, March 2014.

[7] P. P. Bonissone. Soft computing: the convergence of emerging reasoning technologies. Soft Computing- $A$ Fusion of Foundations, Methodologies and Applications, 1(1):6-18, 1997.

[8] A. Brabazon, M. O'Neill, and S. McGarraghy. Natural Computing Algorithms. Natural Computing Series. Springer, 2015. 
[9] C. Cranganu and E. Bautu. Using gene expression programming to estimate sonic log distributions based on the natural gamma ray and deep resistivity logs: a case study from the anadarko basin, oklahoma. Journal of Petroleum Science and Engineering, 70(3):243-255, 2010.

[10] K. Deb, A. Pratap, S. Agarwal, and T. Meyarivan. A fast and elitist multiobjective genetic algorithm: Nsga-ii. Evolutionary Computation, IEEE Transactions on, 6(2):182-197, 2002.

[11] M. Dobróka and N. P. Szabó. Interval inversion of well-logging data for automatic determination of formation boundaries by using a float-encoded genetic algorithm. Journal of Petroleum Science and Engineering, 86:144-152, 2012.

[12] C. Du and J. Cheng. Seismic reservoir fuzzy rules extraction based on ga-bp fnn. In Computational Sciences and Optimization, 2009. CSO 2009. International Joint Conference on, volume 2, pages 850-852. IEEE, 2009.

[13] A. E. Eiben and J. E. Smith. Introduction to evolutionary computing, volume 53. Springer, 2003.

[14] M. Emami Niri and D. E. Lumley. Simultaneous optimization of multiple objective functions for reservoir modeling. Geophysics, 80(5):M53-M67, 2015.

[15] C. Ferreira. Gene expression programming in problem solving. In Soft Computing and Industry, pages 635-653. Springer, 2002.

[16] R. Gholami, A. Moradzadeh, S. Maleki, S. Amiri, and J. Hanachi. Applications of artificial intelligence methods in prediction of permeability in hydrocarbon reservoirs. Journal of Petroleum Science and Engineering, 122:643-656, 2014.

[17] M. S. Hajirahimova. Opportunities and challenges of big data in oil and gas industry. 2015.

[18] A. Hems, A. Soofi, and E. Perez. How innovative oil and gas companies are using big data to outmaneuver the competition, 2013.

[19] H. Kaydani, A. Mohebbi, and A. Baghaie. Permeability prediction based on reservoir zonation by a hybrid neural genetic algorithm in one of the iranian heterogeneous oil reservoirs. Journal of Petroleum Science and Engineering, 78(2):497-504, 2011.

[20] H. Kaydani, A. Mohebbi, and M. Eftekhari. Permeability estimation in heterogeneous oil reservoirs by multi-gene genetic programming algorithm. Journal of Petroleum Science and Engineering, 123:201-206, 2014.

[21] V. Kecman. Learning and soft computing: support vector machines, neural networks, and fuzzy logic models. MIT press, 2001

[22] M. M. Labani, A. Kadkhodaie-Ilkhchi, and K. Salahshoor. Estimation of nmr log parameters from conventional well log data using a committee machine with intelligent systems: a case study from the iranian part of the south pars gas field, persian gulf basin. Journal of Petroleum Science and Engineering, 72(1):175-185, 2010.

[23] J. Leber. Big oil goes mining for big data. MIT Technology Review, May 2012.

[24] H. Li, H. Guo, H. Guo, and Z. Meng. Data mining techniques for complex formation evaluation in petroleum exploration and production: A comparison of feature selection and classification methods. In Computational Intelligence and Industrial Application, 2008. PACIIA'08. Pacific-Asia Workshop on, volume 1, pages 37-43. IEEE, 2008.

[25] X. Li and H. Li. A new method of identification of complex lithologies and reservoirs: task-driven data mining. Journal of Petroleum Science and Engineering, 109:241-249, 2013.

[26] C. Maschio, A. Davolio, M. G. Correia, and D. J. Schiozer. A new framework for geostatistics-based history matching using genetic algorithm with adaptive bounds. Journal of Petroleum Science and Engineering, 127:387-397, 2015.

[27] B. Min, J. M. Kang, S. Chung, C. Park, and I. Jang. Pareto-based multi-objective history matching with respect to individual production performance in a heterogeneous reservoir. Journal of Petroleum Science and Engineering, 122:551-566, 2014.

[28] S. D. Mohaghegh. Reservoir simulation and modeling based on artificial intelligence and data mining (ai\&dm). Journal of Natural Gas Science and Engineering, 3(6):697-705, 2011.

[29] H. Parhizgar, M. R. Dehghani, and A. Eftekhari. Modeling of vaporization enthalpies of petroleum fractions and pure hydrocarbons using genetic programming. Journal of Petroleum Science and Engineering, 112:97-104, 2013.

[30] H.-Y. Park, A. Datta-Gupta, and M. J. King. Handling conflicting multiple objectives using pareto-based evolutionary algorithm during history matching of reservoir performance. Journal of Petroleum Science and Engineering, 125:48-66, 2015.

[31] M. Shaheen, M. Shahbaz, Z. ur Rehman, and A. Guergachi. Data mining applications in hydrocarbon exploration. Artificial Intelligence Review, 35(1):1-18, 2011.

[32] E. M. E.-M. Shokir. Dewpoint pressure model for gas condensate reservoirs based on genetic programming. Energy ${ }^{6}$ Fuels, 22(5):3194-3200, 2008.

[33] D. Xie, D. A. Wilkinson, T. Yu, et al. Permeability estimation using a hybrid genetic programming and fuzzy/neural inference approach. In SPE Annual Technical Conference and Exhibition. Society of Petroleum Engineers, 2005.

[34] Y. Xue, L. Cheng, J. Mou, and W. Zhao. A new fracture prediction method by combining genetic algorithm with neural network in low-permeability reservoirs. Journal of Petroleum Science and Engineering, 121:159-166, 2014.

[35] D. Yong-gang, C. Wei, F. Quantang, and H. Jixiang. Rate decline analysis of fractured gas well by genetic hybrid optimization. In Computational and Information Sciences (ICCIS), 2010 International Conference on, pages 857-860. IEEE, 2010.

[36] L. A. Zadeh. Fuzzy logic, neural networks, and soft computing. Communications of the ACM, 37(3):77-85, 1994.

[37] T. Zhao, V. Jayaram, A. Roy, and K. J. Marfurt. A comparison of classification techniques for seismic facies recognition. Interpretation, 3(4):SAE29-SAE58, 2015. 